\title{
Pseudohypoparathyroidism type 1B - a rare cause of tetany: case report
}

\section{Catarina Garcia, Cátia R. Correia \& Lurdes Lopes}

To cite this article: Catarina Garcia, Cátia R. Correia \& Lurdes Lopes (2017):

Pseudohypoparathyroidism type $1 \mathrm{~B}$ - a rare cause of tetany: case report, Paediatrics and International Child Health, DOI: 10.1080/20469047.2017.1341730

To link to this article: http://dx.doi.org/10.1080/20469047.2017.1341730

册 Published online: 26 Jun 2017.

Submit your article to this journal $\pi$

III Article views: 17

Q View related articles $₫$

View Crossmark data $₫$ 


\title{
Pseudohypoparathyroidism type 1B - a rare cause of tetany: case report
}

\author{
Catarina Garciaa ${ }^{a}$ Cátia R. Correia ${ }^{\mathrm{b}}$ and Lurdes Lopes ${ }^{\mathrm{c}}$ \\ aDepartment of Pediatrics, Hospital Professor Doutor Fernando Fonseca, EPE, Lisbon, Portugal; bepartment of Pediatrics, Centro Hospitalar \\ Lisboa Ocidental, EPE, Lisbon, Portugal; 'Endocrinology Unit, Department of Pediatrics, Centro Hospitalar Lisboa Central, EPE, Lisbon, \\ Portugal
}

\begin{abstract}
Pseudohypoparathyroidism (PHP) is a rare group of disorders characterised by end-organ resistance to the parathyroid hormone (PTH). A 16-year-old boy presented with a 2-year history of involuntary dystonic movements involving mainly the left hand, initially after writing and later during physical exercise. Serum calcium was $1.37 \mathrm{mmol} / \mathrm{L}$ (2.20-2.69), phosphate $2.1 \mathrm{mmol} / \mathrm{L}$ (0.8-1.45) and PTH $302 \mathrm{ng} / \mathrm{L}$ (12-88). CT scan of the head demonstrated multiple subcortical and diffuse basal ganglia calcifications. Genetic analysis confirmed a methylation defect in the GNAS cluster on chromosome 20 q13.32 which established the diagnosis. Treatment with calcitriol and calcium carbonate led to complete remission of symptoms. Causes of hypocalcaemia should be considered in evaluating patients with movement disorders. The diagnosis of PHP-1B is challenging but the overall prognosis is excellent.
\end{abstract}

Abbreviations: PHP: pseudohypoparathyroidism; PTH: parathyroid hormone; PHP1A: pseudohypoparathyroidism type 1A; PHP-1B: pseudohypoparathyroidism type 1B; AHO: Albright's hereditary osteodystrophy; DMRs: different methylated regions; PPHP: pseudopseudohypoparathyroidism

\section{ARTICLE HISTORY}

Received 8 January 2017 Accepted 5 June 2017

\section{KEYWORDS}

Pseudohypoparathyroidism; hypocalcaemia; tetany

\section{Introduction}

Pseudohypoparathyroidism (PHP) is an heterogeneous group of disorders characterised by hypocalcaemia and hyperphosphataemia resulting from end-organ resistance to parathyroid hormone (PTH) [1-9]. It is a rare disease with an estimated prevalence worldwide of approximately 7.9 cases per million $[1,8]$.

The primary effect of PTH on the kidney is to increase calcium absorption from the distal tubules and the thick ascending limb of the loop of Henle and to inhibit phosphate reabsorption at the proximal tubule level. PTH is also responsible for mobilising calcium and phosphate from bone deposits into the circulation [1,2].

In PHP, PTH resistance occurs only at the renal proximal tubule level, representing an unusual form of hormone resistance $[1,9,10]$. The molecular defect responsible for most types of PHP affects the $\alpha$-subunit of the stimulatory G-protein, which is downstream of many different $\mathrm{G}$ protein-coupled hormone receptors, rather than the PTH receptor itself [9-12]. PHP is part of a group of rare diseases associated with genetic disruption of imprinting genes, a phenomenon that restricts gene expression in either the paternally or maternally delivered gene copy [13].
Two main types of PHP have been described: PHP type 1A (PHP-1A) and 1B (PHP-1B). Patients with PHP-1A present with additional hormone resistance (including resistance to thyroid stimulating hormone and gonadotropin) as well as with Albright's hereditary osteodystrophy (AHO), a constellation of features comprising short stature, obesity, round facies, brachydactyly, ectopic ossifications and/or mental retardation $[1,9,10,14]$.

Pseudohypoparathyroidism type 1B is characterised by an absence of AHO features and renal resistance to PTH, and, although initially no resistance to other hormones had been documented, recent reports have shown that other hormonal actions may also be impaired such as thyroid stimulating hormone $[10,15-$ 18]. PHP-1B is most often a sporadic disorder, but sex-influenced autosomal dominant inheritance has been reported [13].

Since parathyroid action is only affected at the renal proximal tubule level, some individuals are asymptomatic and may show only mild elevation of PTH as evidence of hormone resistance $[2,10,11,14]$. However, at some point, most patients present with hypocalcaemia and develop symptoms such as tetany, muscle spasms or even seizures $[2,5,11]$. 

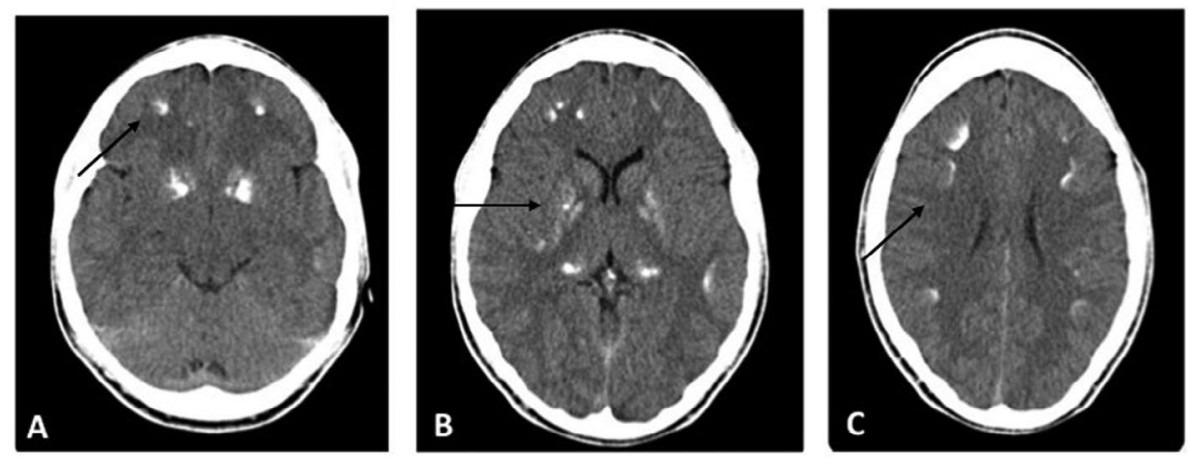

Figure 1. Head CT demonstrating (A) multiple subcortical calcifications in frontal area, (B) basal ganglia and (C) in fronto-parietal areas.

\section{Case report}

A 16-year-old boy was admitted to a general paediatric hospital for evaluation of an intermittent movement disorder. He had been healthy previously, except for a vesicoureteric reflux with no reported urinary tract infections since the age of 2 years. There was no consanguinity and no family history of movement or neurological disorders.

At the age of 14, he developed periods of mild joint pain (mostly in the wrists, knees and elbows) and also episodes of left-hand paralysis while writing, lasting 2-3 min and ceasing spontaneously. Episodes became more frequent during the following 2 years. They occurred mostly during sports, about 20 min after beginning exercise, and were described as a dystonic posture of both hands and forearms. Episodes associated with emotional stress or infections also occurred, also of 3-min duration and with spontaneous cessation. In the month prior to evaluation, he complained of moderate fatigue, weakness and sporadic frontal headaches that responded to analgesics. No aura, dizziness, vertigo, trembling, dysarthria or other symptoms occurred.

For 2 years, no medical care was sought, and when he was 16 he was evaluated at a paediatric hospital. His weight was $51 \mathrm{~kg}$ (10th percentile), height $165 \mathrm{~cm}$ (10th-25th percentile), body mass index of $18.7 \mathrm{~kg} / \mathrm{m}^{2}$ (10th-25th percentile), pubertal development was Tanner stage 5 and physical including neurological examination was normal. Serum calcium was $1.37 \mathrm{mmol} / \mathrm{L}$ (2.20-2.69) and phosphate $2.1 \mathrm{mmol} / \mathrm{L}(0.8-1.45)$.

He was admitted to the Endocrine Unit of Hospital Dona Estefânia, Lisbon for further investigation and treatment.

\section{Investigations}

Haemoglobin, leucocyte and platelet count, liver and kidney function, sodium, potassium, magnesium and chloride levels were normal, as were thyroid function tests. The urinary calcium/creatinine ratio level was also normal. The electrocardiogram demonstrated peaked T-waves and no other abnormalities. Head computed tomography (CT) showed multiple subcortical calcifications in the frontal, parietal and occipital areas as well as diffuse bilateral thalamic and basal ganglia calcifications (Figure 1). Renal ultrasound demonstrated normal cortical-medullar differentiation with bilateral central millimetric lithiasis. The PTH level was $302 \mathrm{ng} / \mathrm{L}$ (12-88), 1,25-dyhydroxyvitamin D level $101.6 \mathrm{pmol} / \mathrm{L}$ (48.9-135.5) and 25-hydroxyvitamin D level $89.25 \mathrm{nmol} / \mathrm{L}$ (50.0-102.5). Because of the clinical suspicion of PHP, genetic analysis was performed which identified a defect in the GNAS cluster on chromosome 20q13.32 with loss of the imprinting at the $A / B$ methylated promoter region. No deletion in the STX16 region (upstream of the GNAS cluster) was detected.

He was commenced on intravenous calcium gluconate and oral calcitriol which was followed by progressive clinical and blood chemistry improvement. Complete remission of symptoms occurred when the total calcium IV dose reached $4.5 \mathrm{~g} /$ day. After commencement of treatment, the patient did not experience any further paroxysmal movements and maintained a calcium level of $2.4 \mathrm{mmol} / \mathrm{L}$ and phosphate level of $1.1 \mathrm{mmol} / \mathrm{L}$ under therapy with calcitriol $(0.75 \mu \mathrm{g} /$ day $)$ and oral calcium carbonate (12 g/day). Laboratory values (PTH, calcium and phosphate levels) returned to normal and a CT scan will be undertaken after 1 year of follow-up.

\section{Discussion}

A 16-year-old adolescent presented with tetany, mostly while practicing sport, and was found to have severe hypocalcaemia and hyperphosphataemia. Genetic analysis demonstrated a methylation defect in the GNAS gene located on chromosome 20q13.32 (this complex locus encodes the $\alpha$-subunit of the heterotrimeric G protein) confirming a diagnosis of PHP type $1 \mathrm{~B}$.

Parathroid hormone, via its Gsa-coupled receptor parathyroid hormone/parathyroid hormone-related peptide receptor (PTHR), is an important regulator of homoeostasis, maintaining blood calcium concentrations within narrow limits. PTH stimulates the release of calcium and phosphate from the bone into the peripheral circulation, acts on the distal nephron increasing 
calcium reabsorption, and in the proximal tubule inhibits phosphate reabsorption and increases 1 - $\alpha$-hydroxylase synthesis. The latter stimulates the synthesis of biologically active 1.25 dihydroxyvitamin $D$, indirectly increasing intestinal calcium reabsorption $[19,20]$.

The hallmark of PHP type $1 \mathrm{~B}$ is the defect in the renal response to PTH owing to the abnormal G-protein function that occurs in renal proximal tubules but not in other sites of PTH action [19]. Defects in this signalling pathway result in PTH resistance and hyperphosphataemia. PTH anticalciuric action occurs in the distal nephron which is not affected in PHP-1B, which might explain why patients have normocalciuria $[9,11]$.

The patient presented with normal urinary calcium levels but the renal ultrasound demonstrated millimetric lithiasis. Although lithiasis is not frequently described in PHP-1B, it is a common feature in other diseases with hyperphosphataemia, e.g. hypothyroidism, since high serum phosphate levels predispose to ectopic mineralisation and consequent kidney stone formation and nephrocalcinosis [21]. Although further studies are necessary, in this patient the elevated serum phosphate might have been responsible for the renal lithiasis. Monitoring the evaluation over time is essential to assess whether regression of the latter is associated with normalisation of phosphate.

Although the genetic mutation is sporadic, it may occasionally have an autosomal dominant transmission pattern. No clinical differences have been reported between the sporadic and the familial forms of PHP-1B [22]. In this report, since the patient had no siblings and the parents had no intention of having another child, no further familial genetic analysis was undertaken.

The heterozygous inactivating mutations involving GNAS exons cause different disorders. This is explained by genomic imprinting, an epigenetic phenomenon affecting a restricted number of genes by which one allele, either maternal or paternal, undergoes a partial or total loss of expression $[9,11,13]$. When the mutation occurs on the maternal allele, PHP-1A patients present with multihormonal resistance and $\mathrm{AHO}$ features. On the other hand, if an inactivating mutation is located on the paternal allele, patients will present with most of the $\mathrm{AHO}$ features but will not be obese or have neurocognitive impairment and usually no hormonal resistance, which is called pseudopseudohypoparathyroidism (PPHP) [23].

Unlike in PHP-1A, PHP-1B patients present with epigenetic defects at one or more DMRs (different methylated regions) of the GNAS gene. Most cases of PHP-1B are sporadic and show loss of methylation at GNAS exons $A / B, X L$ and $A S$. This patient had a loss of imprinting at the $A / B$ methylated promoter region. In the few reports of autosomal dominant PHP-1B, there is a maternal delection either in GNAS or in STX16, which is associated with distinct methylation changes involving different GNAS exons [23].
Despite being congenital, only a few cases of PHP in the neonatal period have been reported [24]. Clinical manifestations typically occur later in childhood, suggesting that PTH resistance and consequent changes in serum calcium and phosphate levels develop gradually. The mechanisms that maintain normal PTH signalling during early infancy remain obscure. Consistent with the literature, no symptoms were evident in this patient before the age of 14 years [14].

Intracranial calcifications, especially in the basal ganglia, have also been described [20]. Usually, these calcifications do not cause clinical complications and sometimes it is discovered accidentally and further investigation leads to the diagnosis of PHP in asymptomatic individuals.

This case demonstrates the importance of considering disorders of calcium metabolism in children presenting with movement abnormalities. In this patient, a dystonic posture was initially associated with repetitive tonic movements such as writing and it was not until 2 years later that he became symptomatic during physical exercise. Causes of hypocalcaemia, including PHP-1B should always be considered in patients with tetany, especially before investigation for a neurological cause of movement disorders.

PHP-1B is a treatable condition and the prognosis is excellent. Patients should be monitored annually for calcium, phosphate, PTH and urinary calcium levels. Although rarely, other endocrinal disorders may be present, so routine screening for abnormal thyroid and gonadotropin function should also be undertaken [14].

\section{Notes on contributors}

Catarina Garcia is a Pediatrics senior resident at the Hospital Prof. Doutor Fernando Fonseca, EPE, Portugal.

Cátia R. Correia is a Pediatrics senior resident at Centro Hospitalar Lisboa Ocidental, EPE, Portugal.

Lurdes Lopes is an Endocrinology Unit coordinator at the Hospital Dona Estefânia, Centro Hospitalar Lisboa Central, EPE, Portugal.

\section{References}

[1] Peres-Nanclares G, Velayos T, Vela A, et al. Pseudohypoparathyroidism type Ib associated with novel duplications in the GNAS locus. PLoS ONE. 2015;10:e0117691.

[2] Nagasaki K, Tsuchiya S, Saitoh A, et al. Neuromuscular symptoms in a patient with familial pseudohypoparathyroidism type lb diagnosed by methylationspecific multiplex ligation-dependent probe amplification. Endocr J. 2013;60:231-236.

[3] Mantovani G, Elli FM. Pseudohypoparathyroidism type 1b in 2015. Ann Endocrinol. 2015;76:101-104.

[4] Root AW, Diamond FB. Disorders of mineral homeostasis in children and adolescents. In: Sperling M, editor. Pediatric Endocrinology. 4th ed. Philadelphia (PA): Elsevier; 2014. p. 734-845. 
[5] GuerreiroR,BrásJ,BatistaS, etal.Pseudohypoparathyroidism type 1-b with neurological involvement is associated with a homozygous PTH1R mutation. Genes Brain Behav. 2016;15:669-677.

[6] Joshi R, Kapdi M. Pseudohypoparathyroidism type $1 \mathrm{~b}$ with hypothyroidism. Indian Pediatr. 2012;49:667-674.

[7] Nakamura A, Hamaguchi E, Horikawa R, et al. Complex genomic rearrangement within the GNAS region associated with familial pseudohypoparathyroidism type 1b. J Clin Endocrinol Metab. 2016;101:2623-2627.

[8] Gómez JL, Momblán JJ, Ibáñez MM, et al. Hypocalcemia in a paediatric case: from the clinical features to diagnosis. J Pediatr Endocr Metabol. 2014;27:139-142.

[9] Mantovani G, Spada A, Elli FM. Pseudohypoparathyroidism and $\mathrm{G}_{\mathrm{s}} \mathrm{\alpha}$-CAMP-linked disorders: current view and open issues. Nat Rev Endocrinol. 2016;12:347-356.

[10] BastepeM.TheGNASlocusandpseudohypoparathyroidism. Adv Exp Med Biol. 2008;626:27-40.

[11] Peres-Nanclares G, Velayos T, Vela A, et al. Pseudohypoparathyroidism type Ib associated with novel duplications in the GNAS locus. PLoS ONE. 2015;10(2):e0117691.

[12] Bastepe M, Juppner H. GNAS locus and pseudohypoparathyroidism. Horm Res. 2005;63:65-74.

[13] Rochtus A, Martin-Trujillo A, Izzi B, et al. Genome-wide DNA methylation analysis of pseudohypoparathyroidism patients with GNAS imprinting defects. Clin Epigenetics. 2016;8:10.

[14] Bastepe M, Jüppner H. Pseudohypoparathyroidism, Albright's hereditary osteodystrophy and progressive osseous heteroplasia. In: Jameson JL, De Groot LJ, editors. Endocrinology: adult and pediatric. 6th ed. Philadelphia (PA): Elsevier Saunders; 2010. p. 1147-1159.

[15] Sano S, Iwara H, Matsubara K, et al. Growth hormone deficiency in monozygotic twins with autosomal dominant pseudohypoparathyroidism type lb. Endocr J. 2015;62:523-529.
[16] Liu J, Erlichman B, Weinstein LS. The stimulatory G protein alpha-subunit $\mathrm{Gs}$ alpha is imprinted in human thyroid glands; implications for thyroid function in pseudohypoparathyroidism types 1A and 1B. J Clin Endocrinol Metab. 2003;88:4336-4341.

[17] Mahmud F, Linglart A, Bastepe $M$, et al. Molecular diagnosis of pseudohypoparathyroidism type $1 \mathrm{~b}$ in a family with presumed paroxysmal dyskinesia. Pediatrics. 2005;115:e242-e244.

[18] Thomas KP, Muthugovindan D, Singer HS. Paroxysmal kinesigenic dyskinesias and pseudohypoparathyroidism type 1b. Pediatr Neurol. 2010;43:61-64.

[19] Gardella TJ, Jüppner H, Brown EM, et al. Parathyroid hormone and the parathyroid hormone receptor type 1 in the regulation of calcium and phosphate homeostasis and bone metabolism. In: Jameson JL, De Groot LJ, editors. Endocrinology: adult and pediatric. 6th ed. Philadelphia (PA): Elsevier Saunders; 2010. p. 969-990.

[20] Mantovani G. Pseudohypoparathyroidism: diagnosis and treatment. J Clin Endocrinol Metab. 2011;96:30203030.

[21] Iglesias P, Bajo MA, Selgas R, et al. Thyroid dysfunction and kidney disease: an update. Rev Endocr Metab Disord. 2017;18:131-144.

[22] Linglart A, Bastepe $M$, Jüppner $H$. Similar clinical and laboratory findingsin patients with symptomaticautosomal dominant and sporadic pseudohypoparathyroidism type $1 \mathrm{~b}$ despite different epigenetic changes in GNAS locus. Clin Endocrinol. 2007;67:822-831.

[23] Tafaj O, Juppner H. Pseudohypoparathyroidism: one gene, several syndromes. J Endocrinol Invest. 2017;40: 347-356.

[24] Narang M, Salota R, Sachdev SS. Neonatal pseudohypoparathyroidism. Indian J Pediatr. 2006;73:97-98. 